\title{
Visualising medical evidence on sepsis treatment
}

\section{A case study about interactive graphics in scientific publishing}

Keywords: science publishing, infographics, data visualisation, health, interactivity, science, publishing, graphics, d3

Visual information can have a range of benefits for busy health practitioners. At The BMJ, we often use visuals to provide quick summaries of information from at times lengthy articles. This paper presents a case study of the design process for an interactive graphic on sepsis treatment, aiming to update doctors working in intensive care units of current evidence. Through explaining this design process, four major challenges for the use of interactive graphics in science publishing are highlighted: (1) how to identify suitable knowledge for visualisations; (2) how to select an appropriate depth of information for a particular project; (3) how interactive graphics can be a permanent part of the scientific record, and (4) how they can be deployed across a range of platforms and devices.

\section{Infographics at The BMJ}

The BMJ (formerly the British Medical Journal) is a respected medical research publisher, with a citation rate among the top five general medical journals in the world.
Unusually for a scientific journal, it also publishes educational content, news and comments, aimed at informing medical practitioners. However, doctors, clinicians and other medical professionals are increasingly busy, and therefore find it hard to keep up with the volume of scientific evidence that is published (Sackett 1996: 71).

Data visualisation and infographics, which often summarise key parts of articles, can help people to quickly understand information (Resnikoff 1989). They can also help people to visualise uncertainty (Spiegelhalter, Pearson \& Short 2011), which is often useful when applying health research to medical practice.

To explore the benefits of information visualisation in these areas, I have been serving as the journal's first data graphics designer since 2014. My remit is to visualise data and information from the papers we publish, making it quicker and easier for time-pressured doctors to access them. As of writing, around 60 infographics projects have been completed, all of which are available on The BMJ website (The BMJ, Infographics 2017).

Publishing infographics in a scientific journal, particularly interactive ones, can be a challenging process. This case study describes the design process of an infographic on treatments for sepsis to highlight some of the challenges inherent in publishing interactive visual materials within scientific journals. 


\section{Initial development}

The BMJ publishes several hundred articles a year (The BMJ, Resources for Authors 2016). Many of these articles are lengthy and complex, thus identifying suitable topics for visualisation can be challenging. Specialist medical knowledge is of course essential, as well as design and editorial experience. It is common in publishing for authors, editors and designers to work sequentially, but in the case of publishing health infographics in The $\mathrm{BMJ}$, the interaction between these professionals is more closely intertwined.

In the case of the sepsis graphic, the paper it was to accompany was one of The BMJ's 'State of the Art' reviews, which are lengthy and detailed papers designed to summarise current evidence in a particular field (Groves 2014). The originally submitted manuscript for the paper was 69 pages long, detailing risk factors, causes, cellular/molecular pathways, and evidence for different treatment strategies in sepsis.

The first step was to identify information and themes that could be relevant to a practitioner. This process involved the designer, the section editor (also a general medical practitioner), and the authors (from a US research institute). The discussions aimed at finding useful information which could be presented in a concise visual layout. The four sketches developed to facilitate the decision are provided in Figure 1.

Idea a presents a timeline of evidence, showing which studies have since been superseded, and which are still current.

Idea $\mathbf{b}$ presents a similar timeline at the top, but focusses more on categorising treatments, grouping together all studies on fluid management, different kinds of drugs, and so on. Note the different layouts for desktop and mobile.
Idea c shows how invasive treatments in intensive care units, such as intravenous fluids, can be routes for further infections to enter the body.

Idea $\mathbf{d}$ gives another summary of evidence for treatments, but this time centres on an image of a patient in an intensive care unit.

To decide which of these ideas was likely to strike a chord with our readers, we needed to weigh up the time needed to read the graphic, the visual appeal, and the usefulness of the information contained. Other considerations that had to be factored in were the resources available (design time and artwork budget), and the project timeline (so that the graphic could be published together with the article).

Our main guiding principle in making such decisions is the "vision" of BMJ Publishing Ltd: "to create a healthier world" (BMJ, Brand proposition and purpose statement 2017). Following this principle, we try to focus on graphics which have an obvious and practical application to improving healthcare. To help us make informed decisions about which graphics have real benefits, we often show graphics to junior doctors who spend time visiting the journal on a weekly rotation, or to our clinical editors, who are themselves practising doctors, since it is important to involve the users when making such decisions (Sanders \& Stappers 2008).

In this case, we felt that idea "d" had a good combination of visual interest with concise, useful advice that could be directly put into practice. It was also important that the project could be completed in the time available.

\section{Visual elements}

The next stage in our design process is to produce a static (non-interactive) version which looks as close 
(a)

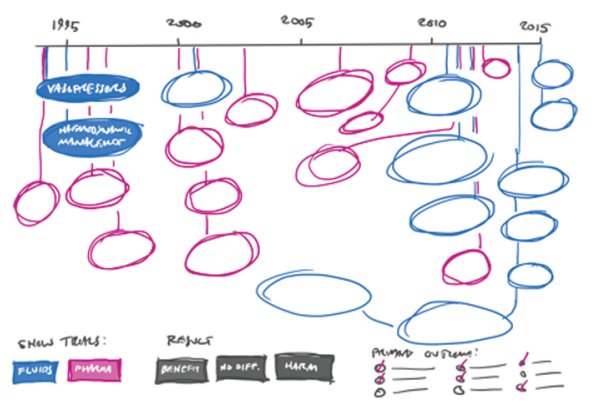

(c)

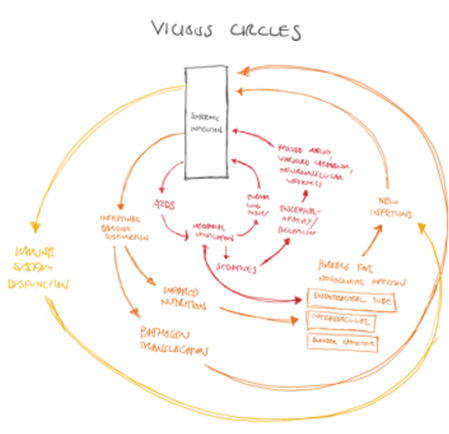

(b)

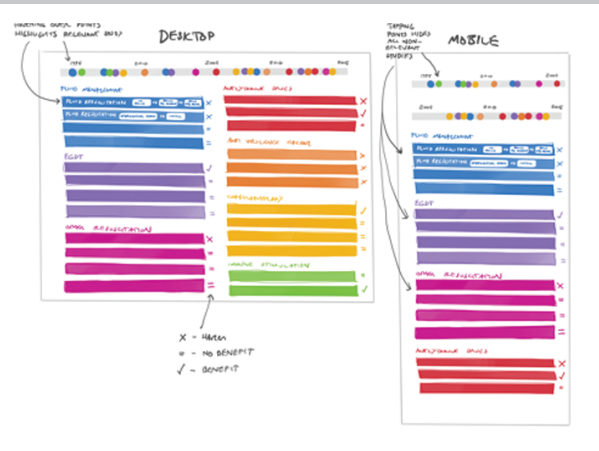

(d)

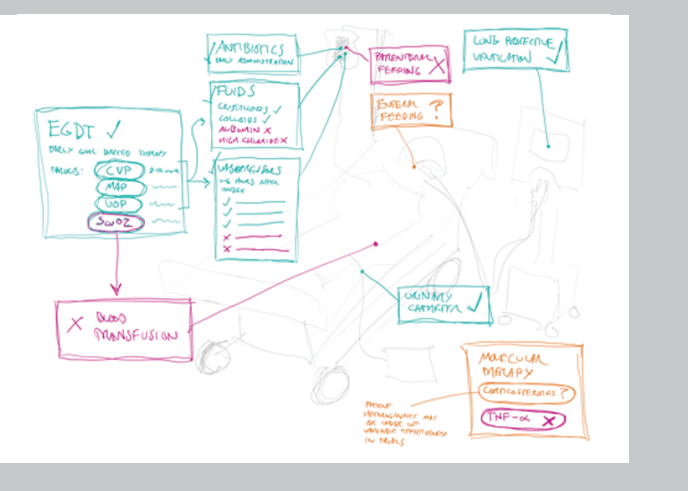

Figure 1. Four different sketches for a graphic about sepsis: (a) timeline of published studies; (b) timeline and categorisation of published studies; (c) illustrating the cyclical nature of invasive treatment and infection routes; (d) presenting evidence for treatments over an illustration of a sepsis patient.

to the final graphic as possible. In this case, we could not find a stock image or illustration that had the level of detail required for the graphic's central image, so we commissioned an illustration from the KJA Artists Agency (KJA Artists 2017). The rough (Figure 2a) was checked by our clinical editors, who asked for a number of changes: an opaque cover over the feeding bag, a monitor displaying information on the wall, and raised cot sides (Figure $2 \mathrm{~b}$ ). The final artwork can be seen in Figure 3.
Once the illustration was approved, it was incorporated into a static layout, produced using Adobe Illustrator (Figure 4). This layout introduced the boxes that show whether the treatments are supported by evidence (green), are uncertain (purple) or not recommended (pink). We considered using the same perspective and vanishing point as the artwork for the treatment information (Figure 4a) but decided that the long drug names in the "Vasopressors" box were too difficult to read, compared with a flatter layout (Figure $4 \mathrm{~b}$ ). 


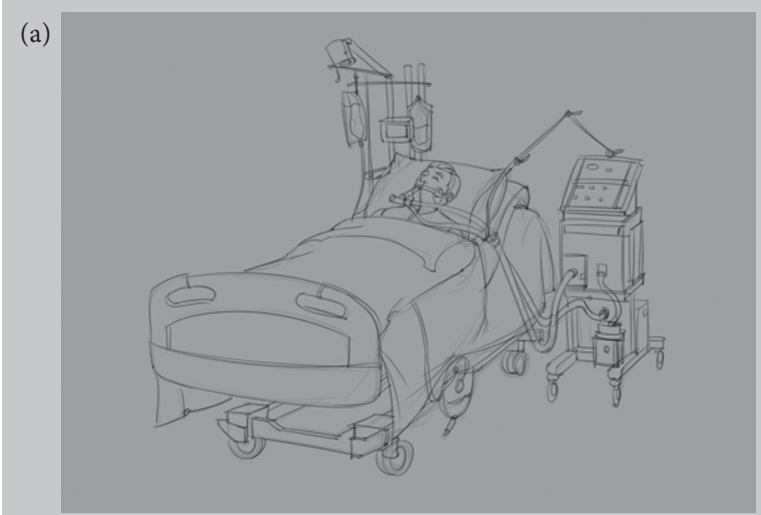

(b)

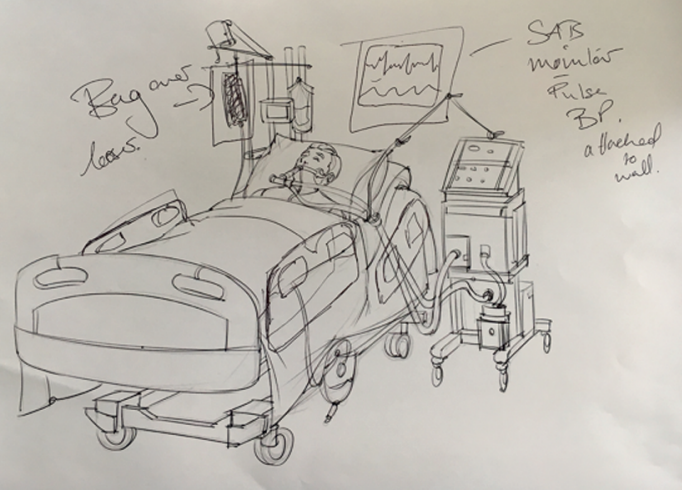

Figure 2. Artwork roughs: (a) is the original provided by the illustrator; (b) includes the additions made by the journal's clinical editors.

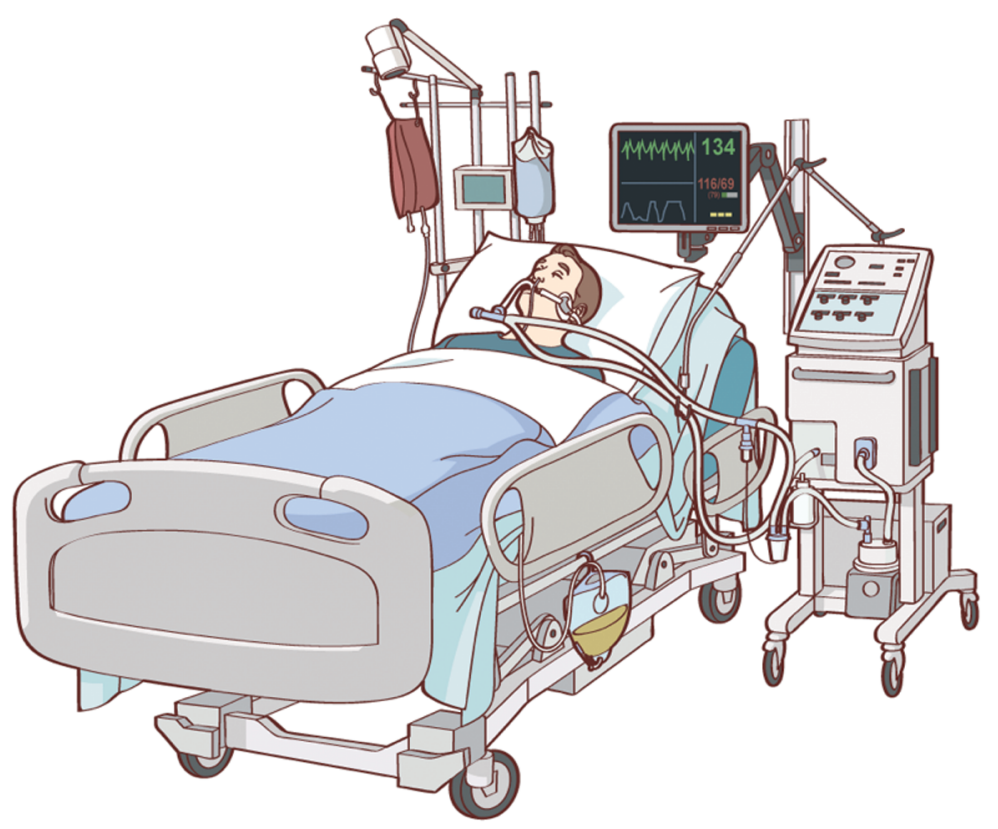

Figure 3. Final artwork: showing a patient in an intensive care unit, with the treatments that would be needed for sepsis. 
(a)

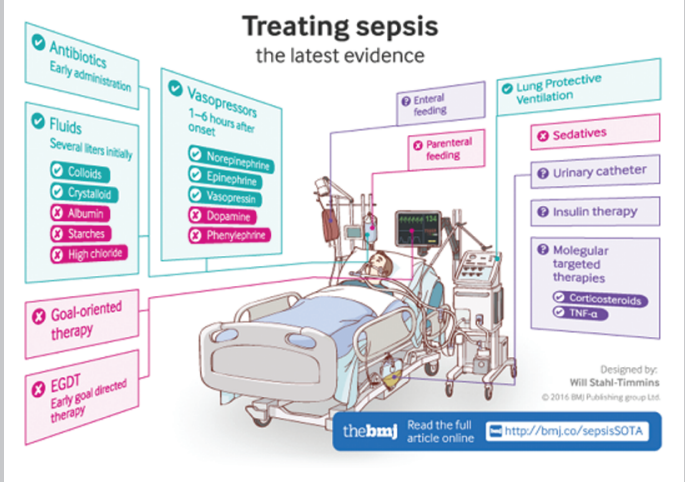

(b)

Treating sepsis: the latest evidence

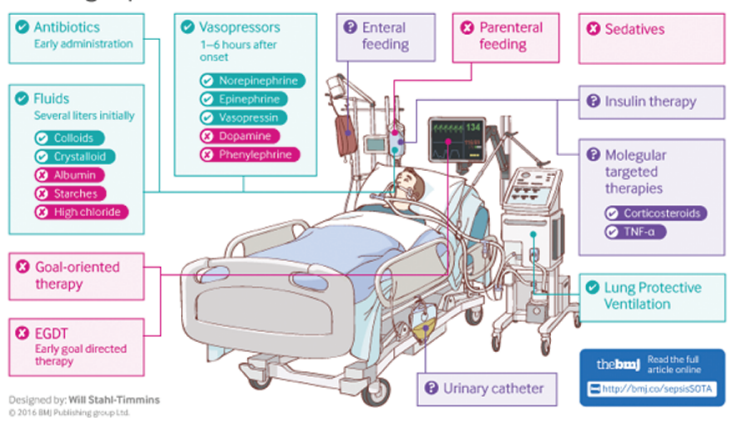

Figure 4. Two alternative layouts.

\section{Coding interactivity}

The final stage of the design process involved coding the interactive elements. The user interface is a very simple one: Once the user selects a box, further information on that treatment is revealed. However, to create even this basic functionality requires some effort-particularly when considering that it must work within a responsive page layout. It must work equally well on different sized devices with different input methods, different browser versions. It may also be accessed by people using screen reading software, or automated systems. To allow that much information to be as machine readable as possible, the final piece consists of four layers:

1. The bottom layer contains the illustration of the sepsis patient, in PNG image format (Figure 5a).

2. The second layer contains the coloured boxes. When the boxes are selected, they bring up more detailed information on each treatment (Figure $5 \mathrm{~d}$ ). The code to draw these boxes is written in javascript, making use of the $\mathrm{d}_{3}$.js library ${ }^{1}$ (Figure 5e).

3. The next layer appears overlaid above the other two, and is a static SVG (scaleable vector graphics) layer, containing the box labels and arrows (Figure 5b).

4. The very top layer is the pop-up boxes that display when one of the boxes in layer 2 is selected (Figure 5d). These are stored on the website as separate SVG files.

This way of structuring the graphic allows it to be easily scaled to different screen sizes, and for the majority of the content within the graphic (everything except the background image) to be machine readable. However, as we will discuss later, there are potential problems with future-proofing such interactive pieces, since to interpret them, a number of different standards and libraries are needed-it is dependent on the user's device being able to interpret particular versions of HTML, CSS, Javascript, SVG, the d3.js library, and PNG images. 
(a)

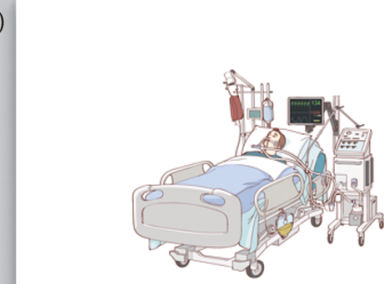

(d)

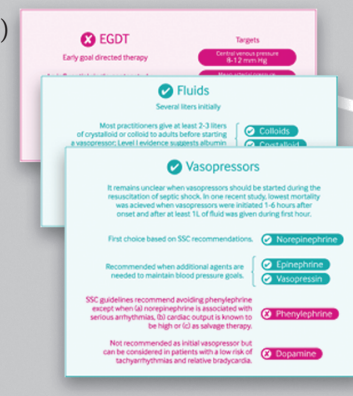

(b)
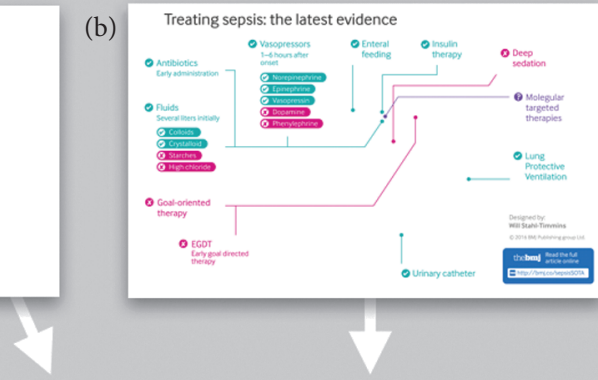

(f)

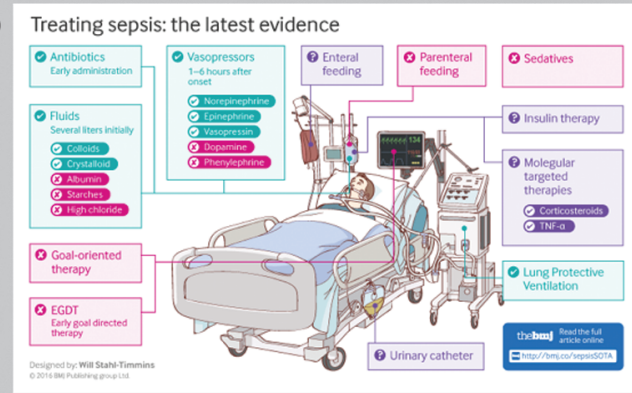

(c)

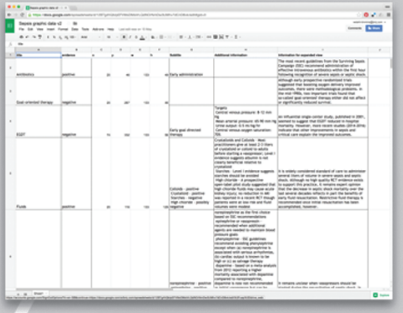

(e)

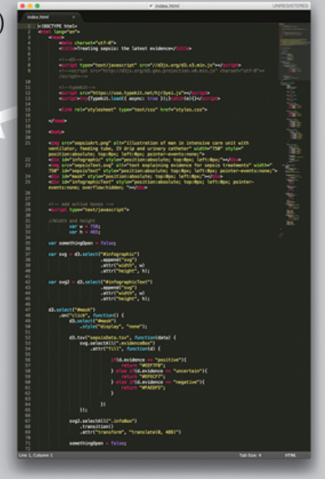

Figure 5. The component parts of the interactive graphic: (a) background image (PNG format); (b) labels and arrows (SVG format); (c) data file with x/y positions, sizes, and colours of clickable boxes (TSV format); (d) information pop-ups (SVG format); (e) code for interactivity (HTML, CSS and javascript formats); (f) final appearance of graphic on loading.

After the design process was finished, the graphic entered our production process. It was checked by our in-house editors and proofreaders for style and consistency before deployment. With potentially life-saving treatment information such as this, it is vital to ensure that no errors have been introduced during the design process. The graphic was also tested for functionality on a range of different browsers and operating systems. It was published in May 2016, and is available on The BMJ website. ${ }^{2}$

\section{Challenges}

The development and publishing process of this graphic highlights four challenges inherent in designing interactive graphics for scientific publications:

1. How to identify suitable knowledge for visualisations

2. How to select an appropriate depth of information for a particular project

3. How interactive graphics can be a permanent part of the scientific record 
4. How interactive graphics can be deployed across a range of platforms and devices

\subsection{How to identify suitable knowledge for visualisations}

As suggested at the start of the case study, it is challenging to identify appropriate articles and information that are suitable for visualisations. The role of Marie Neurath in the Isotype Institute, that of the transformer (Macdonald-Ross \& Waller 2000: 177), is a valuable one in information design, and one that is shared between designers and editors at The BMJ. It may prove, in time, that to have one person devoted to mediating between editors and designers, and to sourcing and identifying suitable information for visualisations could be a good solution to the problem of overwhelming volume of information.

\subsection{How to select an appropriate depth of information for a particular project}

It is also challenging to decide what the right amount of information for an interactive graphic or visualization is. Some of our graphics have no more than 6 or 7 data points, and are designed to provoke a quick reaction on social media. Others have many thousands, and are designed for much longer and deeper engagements. The decision about how much information to present is likely to be based on the underlying articles, but it is also helpful to involve readers and users of graphics in this kind of decision. Interactive graphics have the advantage that not all information needs to be presented to all users, but careful selection of which information to include is still important, so as to prevent the reader from feeling lost, or unable to find the information they require.

\subsection{How interactive graphics can be a permanent part of the scientific record}

The BMJ's articles, at the time at which they are published, become part of the scientific record. They are indexed by central databases such as PubMed and Web of Science. These platforms are designed to store text articles with figures, but not interactive infographics. There are also problems around archiving this information. When javascript is updated, or the libraries are used for infographics such as $\mathrm{d}_{3}$.js, there is no guarantee that graphics produced in the past will continue to work as intended (or at all). The BMJ's current strategy is to provide these databases with a PDF file showing a static screenshot of the interactive, and URL that shows where that graphic can be found on The BMJ's website. While The BMJ has been published since the 1840 s, and it is not planning to close, the question remains what would happen if it did shut down. While some information is contained in both graphics and the accompanying paper, it is in a different format in the graphic-the visual elements can provide a different view of the information in a non-linear format for example, or allow different connections to be made between information that would not be possible in text alone.

\subsection{How interactive graphics can be deployed across a range of platforms and devices}

The BMJ's infographics are currently viewed on computer screens, tablets and smartphones-devices with screens in different sizes, and with different input methods. In this case, the graphic is simple enough that the viewer can be expected to pan and zoom as necessary on a small screen to navigate the information, although the large pop-up boxes are not particularly user-friendly if zoomed too far in. We have also experimented with 
graphics which reorder themselves to fit the size of the screen (The BMJ, Zika: A clinical guide 2016). However, this tends to limit us to one single design: Separate boxes with information in them. For information that is complex and requires interrelated components, such layouts are often not the most effective ones. It remains challenging to address the needs of different people using graphics in all kinds of places, from a quiet office with a large screen to a busy hospital ward with a smartphone.

\section{Conclusion}

The BMJ's infographics continue to offer many different ways of presenting information. We are currently experimenting with new production methods, such as automating the creation of infographics to accompany particular article types, so that a greater volume of visual material can be produced for our readers. But challenges remain in terms of how to identify and organise suitable information for visualisations, and how to distribute and record interactive, visual artefacts. There is a need for new methods of indexing and archiving interactive graphics in scientific journals.

Submission date: 7 October, 2016

Accepted date: 8 February, 2017

\section{Notes}

1. Described on the d3 website as: "a JavaScript library for manipulating documents based on data" (see https://d3js.org/).

2. URL for accessing infographic: http://www.bmj.com/content/ 353/bmj.i1585

\section{References}

BMJ. (2017) Brand proposition and purpose statement. http:// www.bmj.com/company/bmj-brand-guidelines/brandproposition-and-purpose-statement/ [accessed o4 Feb 2017].

Groves, T. (2014) State of the art, The BMJ, 348:91388. doi: 10.1136/bmj.g1388

KJA Artists, http://www.kja-artists.com/ [accessed o3 Feb 2017].

Macdonald-Ross, M. \& Waller, R. (2000) The Transformer Revisited, Information Design Journal, 9(2/3), 177-193. doi: 10.1075/idj.9.2-3.06mac

Resnikoff, H.L. (1989) The illusion of reality. London, UK: Springer. doi: 10.1007/978-1-4612-3474-6

Sackett, D.L. (1996) Evidence based medicine: what it is and what it isn't, The BMJ, 312, 71.

Sanders, E.B.N. \& Stappers, P.J. (2008) Co-creation and the new landscapes of design, CoDesign: International Journal of CoCreation in Design and the Arts, 4(1), 5-18.

Spiegelhalter, D., Pearson, M. \& Short, I. (2011) Visualizing Uncertainty About the Future, Science, 333, 1393. doi: $10.1126 /$ science.1191181

The BMJ, Infographics, http://www.bmj.com/infographics [accessed 03 Feb 2017].

The BMJ, Resources for Authors, http://www.bmj.com/about-bmj/ resources-authors [accessed 7th Oct 2016].

The BMJ, Zika: A clinical guide, http://sandpit.bmj.com/graphics/2016/zika/index.html [accessed o3 Feb 2017].

\section{About the author}

Will Stahl-Timmins is an information graphic designer working for The BMJ, creating visualisations for doctors and researchers. He believes that visualising data can help to transform it into something much more valuable: knowledge. His career has focussed

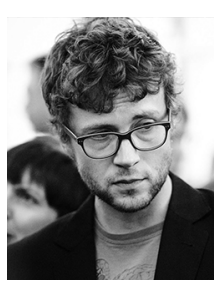
on visual communication of health research, using infographics and data visualisation techniques. He turns raw data into engaging visual stories, so that the information can immediately be put to use by those who need it.

Email: wstahl-timmins@bmj.com 